\title{
Evaluation of clinical effectiveness of paclitaxel and ursolic acid co-loaded liposomes as enhanced treatment for head and neck squamous cell carcinoma
}

\author{
Bi-Hua Lv', Wei Tan ${ }^{2}$, Xuejun Shang ${ }^{3}$, Ling Zhang ${ }^{4 \star}$ \\ ${ }^{1}$ Department of Pharmacy, Zhongnan Hospital of Wuhan University, Wuhan, Hubein 430071, ${ }^{2}$ Department of Radiology, \\ Hospital of Wuhan University of Science and Technology, Wuhan, 430081, ${ }^{3}$ Department of Andrology, Jinling Hospital Affiliated \\ to Nanjing University School of Medicine, Nanjing 210002, ${ }^{4}$ Department of Environmental Hygiene and Occupational Medicine, \\ School of Public Health, Hubei Province Key Laboratory of Occupational Hazard Identification and Control, Wuhan University of \\ Science and Technology, Wuhan 430081, China
}

*For correspondence: Email: zhangling@wust.edu.cn, shangxj98@163.com

Sent for review: 20 July 2018

Revised accepted: 30 October 2018

\begin{abstract}
Purpose: To enhance the clinical effectiveness of paclitaxel (PTX) by co-delivery with ursolic acid (UA) for the treatment of head and neck cancer

Methods: Co-loaded liposomes of PTX and UA (UA-PTX-LiP) were prepared by thin-film hydration method. Their size and loading efficiency were determined using dynamic light scattering (DLS) technique and high performance liquid chromatography (HPLC), respectively. The effectiveness of UAPTX-LiP against HSC-3 human head and neck cancer cell-lines was compared with that of PTX liposome (PTX-LiP) using systemic cell-based in vitro evaluation with MTT assay. Fluorescent microscopy was used for cell uptake studies.

Results: The size of the prepared UA-PTX-LiP was $126.5 \pm 3.22 \mathrm{~nm}$. The ratiometric system for PTX and $U A$ as liposomes revealed significantly enhanced cytotoxicity, with comparatively lower $I C_{50}$, when compared to individual PTX-Lip. Fluorescent microscopy revealed the internalization ability of UA-PTXLiP by targeted delivery of PTX in HSC-3 human head and neck cancer cell-line.

Conclusion: These results show that UA-PTX-LiP successfully enhances the therapeutic potential and clinical outcomes of PTX in head-and-neck cancer, and also demonstrate the useful effect of combination of UA and PTX in chemotherapy.
\end{abstract}

Keywords: Paclitaxel, Ursolic acid, Combination chemotherapy, Head-and-neck squamous cancer

\begin{abstract}
This is an Open Access article that uses a funding model which does not charge readers or their institutions for access and distributed under the terms of the Creative Commons Attribution License (http://creativecommons.org/licenses/by/4.0) and the Budapest Open Access Initiative (http://www.budapestopenaccessinitiative.org/read), which permit unrestricted use, distribution, and reproduction in any medium, provided the original work is properly credited.

Tropical Journal of Pharmaceutical Research is indexed by Science Citation Index (SciSearch), Scopus, International Pharmaceutical Abstract, Chemical Abstracts, Embase, Index Copernicus, EBSCO, African Index Medicus, JournalSeek, Journal Citation Reports/Science Edition, Directory of Open Access Journals (DOAJ), African Journal Online, Bioline International, Open-J-Gate and Pharmacy Abstracts
\end{abstract}

\section{INTRODUCTION}

Head-and-neck cancer is ranked amongst the most fatal cancers owing to its poor prognosis. Squamous cell carcinoma of the head and neck (HNSCC) proliferates at the brink of oral cavity, paranasal sinuses and nasal cavity along with larynx and pharynx, and contributes to nearly 900,000 new cases and 350,000 mortalities annually worldwide [1]. Paclitaxel (PTX) is one of the most valued anticancer drugs for the treatment of breast, colon, prostate, melanoma 
and various types of solid cancers [2]. However, it has some drawbacks such as poor aqueous solubility, gastrointestinal toxicity and nonspecific systemic delivery [3]. Ursolic acid (UA) isolated from the roots of Catharanthus trichophyllus possesses anti-tumor and antiinflammatory properties [4].

The anticancer properties of PTX and UA can be employed for synergistic combination therapy of HNSCC. The inception of novel drug delivery systems has enhanced the anticancer potential of drugs $[5,6]$. Therefore, in this study, lipid bilayer liposomes (LiP) that can efficiently codeliver both hydrophobic and hydrophilic drugs by co-loading them in their hydrophilic core and hydrophobic lipid vesicles were chosen as for the co-delivery of PTX and UA $[5,7,8]$. In a recent investigation, it was shown that hybrid nanoconstruct of albumin-PTX hybrid nanoparticle (LAPN) encapsulated in liposome significantly enhanced anticancer effectiveness by increasing the accumulation of PTX in tumor, bio-distribution and pharmacokinetic parameters [9].

Studies have shown that UA attenuates colon cancer by increasing cancer cell apoptosis, and also by arresting angiogenesis through inhibition of the expressions of COX-2 and caspases [1012]. In the present study, liposomes incorporating both PTX and UA (UA-PTX-LiP) were designed for efficient co-delivery of the two drugs. The resultant UA-PTX-LiP was inspected thoroughly to ensure that it is an effective co-delivery system for combination chemotherapy [13].

The objective of this study was to develop UAPTX-LiP formulation for the co-delivery of UA and PTX for effective chemotherapy, based on a hypothesis that the combination of UA and PTX could enhance the therapeutic potency of PTX for effective treatment of cancer.

\section{EXPERIMENTAL}

\section{Materials}

Paclitaxel (PTX) was purchased from Hefei Bo Mei Biotechnology Co., Ltd. China, while UA was procured from Beijing Huafeng United Technology Co., Ltd. (Beijing, China). HSPC and DSPE-PEG2000 were acquired from Avanti Polar Lipids, Inc; 3-(4, 5-dimethylthiazol-2-yl)-2, 5-diphenyl tetrazolium bromide (MTT) was obtained from Sigma Aldrich (St. Louis, MO). All other chemicals used for experiments were of analytical grade. Milli-Q water (Milli-Q plus 185 purification system, Bedford, MA)) was used for all aqueous preparations throughout the study.

\section{Preparation of PTX and UA-PTX liposomes}

The liposomes were prepared using the thin-film dispersion hydration method to formulate liposomes co-loaded with PTX or UA/PTX [14]. The lipid compositions were HSPC/DSPEPEG2000/Chol at a molar ratio of $65: 9: 26$. The PTX- or UA-PTX-to-lipid ratio was 1:20 (w/w), and the UA:PTX ratio was kept at 1:1 (w/w). The PTX or UA-PTX and lipids were dissolved in chloroform, and the lipid dispersion was dried in a rotary evaporator under reduced pressure at 45 ${ }^{\circ} \mathrm{C}$ to constitute a thin lipid layer. The thin layer of lipid was then hydrated using $4 \mathrm{ml}$ of PBS at $\mathrm{pH}$ 6.8 , and incubated for $30 \mathrm{~min}$ at $50^{\circ} \mathrm{C}$. The resultant lipid vesicles were extruded through polycarbonate membrane (200 nm pore size) 5 times to form homogeneous unilamellar vesicles with aid of a Lipex Extruder driven by pressurized nitrogen at $60^{\circ} \mathrm{C}$. The liposomes were washed and lyophilized to obtain PTX-Lip and UA-PTXLiP.

\section{Physicochemical characterization of PTX-LiP and UA-PTX-LiP}

\section{Particle characterization}

The PTX-LiP and UA-PTX-Lip were evaluated with respect to mean particle size, size distribution and zeta potential by means of Malvern Zetasizer Nano ZS (Malvern 3000HS, France). All measurements were repeated thrice [15].

\section{Transmission electron microscopy (TEM)}

Freshly-prepared UA-PTX-LiP was spread on a carbon-coated copper grid to form a thin liquid film. The film was stained with $1 \%$ (w/v) phosphotungstic. Excess staining solution was cleared with filter paper and then the film was allowed to air-dry. The stained film was photographed using a transmission electron microscope.

\section{High performance liquid chromatography (HPLC)}

Following liposome lysis, methanol was used to extract PTX and UA which were quantified using HPLC analysis at maximum absorption wavelengths of $227 \mathrm{~nm}$ and $210 \mathrm{~nm}$, respectively [16]. The filtered sample $(20 \mu \mathrm{L})$ was introduced through the injector port into a C18 column (250 $\mathrm{mm}, 4.6 \mathrm{~mm}, 5 \mu \mathrm{m}$; Merck). The mobile phase used for simultaneous estimation was 55:45 (v: v) mixture of methanol and acetonitrile at a flow rate of $1.0 \mathrm{ml} / \mathrm{min}$. 


\section{Entrapment efficiency (EE) and drug loading (DL)}

Entrapment efficiency (EE, \%) was estimated by analysing PTX and UA levels in PTX-LiP and UA-PTX-Lip using HPLC method as described above. The liposomes were subjected to lowspeed centrifugation at $3000 \mathrm{~g}$ for $10 \mathrm{~min}$ at room temperature to separate the un-entrapped UA and PTX dispersed in the supernatant liquid. The supernatant was filtered and used for evaluation of free UA and PTX [17].

EE and DL were calculated as in Eqs 1 - 4.

$$
\begin{aligned}
& E E(\%)=\frac{W 1}{W 2} \times 100 \ldots \ldots \ldots(1) \text { for PTX } \\
& E E(\%)=\frac{w s}{w a} \times 100 \ldots \ldots \ldots(2) \text { for } U A \\
& D L(\%)=\frac{w 1}{w 5} \times 100 \ldots \ldots \ldots(3) \quad \text { for } P T X \\
& D L(\%)=\frac{w 2}{w 5} \times 100 \ldots \ldots \ldots(4) \quad \text { for UA }
\end{aligned}
$$

where W1 is the weight of PTX in PTX-LiP; W2 is the weight of PTX initially taken; $W 3$ is the weight of UA in UA-PTX-LiP; W4 is the weight of UA initially taken; W5 is the weight of PTX-LiP, and W6 is the weight of UA-PTX-LiP.

\section{Cellular uptake studies}

The cellular uptakes of PTX-LiP and UA-PTX-LiP formulations were determined in HSC-3 cells so as to appraise drug accumulation in the cancer cells. The internalization of PTX-LiP and UAPTX-LiP in HSC-3 human head and neck cancer cell-lines was evaluated using FACS. The HSC-3 human head-and-neck cancer cells were cultured in McCoy's 5A media (Sigma Aldrich) supplemented with $10 \%$ fetal bovine serum (Gibco BRL), antibiotics and L-glutamine at 37 ${ }^{\circ} \mathrm{C}$. Fluorescein isothiocyanate (FITC)-loaded PTX-LiP (FITC-LiP) and FITC-loaded UA-PTXLiP (UA-FITC-LiP), were prepared by adding $1 \%$ $(w / v)$ FITC in PBS used for hydration of thin lipid layer during the preparation of PTX-LiP and UAPTX-LiP.

All the procedures were performed in the dark. The HSC-3 cells were seeded in 6-well plates using fresh culture media, and incubated for 24 h. Thereafter, FITC-LiP and UA-FITC-LiP were added to the culture plates and incubated for $4 \mathrm{~h}$, after which the cells were washed thrice with PBS and processed for flow cytometric analysis at an excitation wavelength of $480 \mathrm{~nm}$ and an emission wavelength of $550 \mathrm{~nm}$.

\section{Apoptosis assay}

Annexin V/PI double stain assay was used for determination of apoptosis of HSC-3 cells in 12well plates. The cells were incubated up to $24 \mathrm{~h}$ so that they attained adherence at the bottom. Then, the medium was changed for a fresh one, after which the cells were treated separately with control, PTX-LiP and UA-PTX-LiP, and again incubated for $24 \mathrm{~h}$. Following the incubation, the HSC-3 cells were washed thrice with PBS, followed by trypsinization and cell harvesting. The collected cells were subjected to centrifugation for $5 \mathrm{~min}$ at $1500 \mathrm{rpm}$ and suspended in $500 \mu \mathrm{l}$ of PBS. Finally, the cells were suspended in binding buffer cocktail, double-stained with FITC-Annexin V and PI, and incubated in the dark for $15 \mathrm{~min}$. The cells were immediately analysed using BD FACS Calibre flow cytometer.

\section{Determination of intracellular reactive oxygen species (ROS)}

The intracellular production of ROS was determined using 2', 7'-dichlorofluorescin diacetate (DCFDA), based on the principle that on oxidation, DCFDA is de-esterified inside the cell matrix into a highly fluorescent $2^{\prime}, 7^{\prime}$ dichlorofluorescein (DCF). The HSC-3 cells were cultured in a 6-well plate and incubated for $24 \mathrm{~h}$. Then, the cells were treated with UA-LiP, UAPTX-LiP and blank liposomes for the indicated durations at $37{ }^{\circ} \mathrm{C}$ in a $5 \% \mathrm{CO}_{2}$ incubator, after which they were flushed with PBS and incubated with $5 \mu \mathrm{M}$ of DCFDA in the dark for $30 \mathrm{~min}$. Then, the cells were washed again with PBS and reconstituted in $500 \mu \mathrm{l}$ of PBS. Thereafter, the levels of cellular ROS were determined using fluorescence-activated cell sorter (FACS, Calibur, BD Biosciences, USA). The DCF data were recorded at an excitation wavelength of 488 $\mathrm{nm}$ and emission wavelengths of $515-540 \mathrm{~nm}$.

\section{Cytotoxicity studies}

In vitro cytotoxicities of PTX, PTX-LiP and UAPTX-LiP were determined in HSC-3 cells using MTT assay [18]. The HSC-3 cells were cultured and maintained as described in previous sections. Briefly, the cells were seeded at a density of 5000 cells/well in 96 -well plates for 24 h. Then, the cells were treated with PTX, PTXLiP and UA-PTX-LiP at comparable concentrations of PTX, i.e. $0.001,0.01,0.1,1$ and $10 \mu \mathrm{g} / \mathrm{ml}$, followed by incubation for $24 \mathrm{~h}$. Thereafter, MTT solution was added, and the cells were further incubated for $3 \mathrm{~h}$. After discarding culture medium from each well, the resultant formazan crystals were dissolved in $\mathbf{1 5 0}$ 
$\mu \mathrm{l}$ of DMSO, and the absorbance of each solution was measured in a Bio-Rad 680 micro plate reader at $570 \mathrm{~nm}$. The experiments were performed in triplicate, and cell viability (V) was calculated as in Eq 5.

$\mathrm{V}=(\mathrm{At} / \mathrm{Ac}) 100$

where At and Ac are the absorbance of treated and control groups, respectively.

\section{Lactate dehydrogenase (LDH) release assay}

The HSC-3 cells were seeded in 96-well plate for $24 \mathrm{~h}$ at a density of $1 \times 10^{4}$ cells per well. Then, the cells were treated with PTX, PTX-LiP and UA-PTX-LiP at equivalent concentration of $1 \mu \mathrm{g} / \mathrm{mL}$. After $24 \mathrm{~h}$, LDH assay was performed as specified in the manufacturer's protocol [19]. In this assay, $1 \mathrm{~mL}$ of $3 \%$ Triton $X 100$ and $1 \mathrm{~mL}$ PBS ( $\mathrm{pH} 7.4)$ were used as positive and negative controls, respectively. The level of $\mathrm{LDH}$ released was used as a marker of toxicity [20].

\section{Statistical analysis}

Graph Pad Instat software (Graph Pad Software Inc., CA, USA) was used for one-way analysis of variance (ANOVA), followed by Turkey-Kramer test for comparing multiple tests. The significance level was fixed at $p<0.05$. Values of $I_{50}$ were calculated using Prism 5 software.

\section{RESULTS}

\section{Characteristics of PTX-LiP and UA-PTX-LiP}

The mean sizes of PTX-LiP and UA-PTX-LiP were $109.7 \pm 2.11$ and $126.5 \pm 3.22 \mathrm{~nm}$, respectively. The zeta potentials of PTX-LiP and UA-PTX-LiP were $-24.9 \pm 1.22$ and $-26.4 \pm 2.11$ $\mathrm{mV}$, respectively. Their zeta potential values were negative, which might be due to their phospholipid contents [21]. The encapsulation efficiency (EE) and drug loading of PTX were 97 and $5.7 \%$ in UA-PTX-LiP, and 89 and $6.2 \%$ in PTX-LiP, respectively. These results are presented in Table 1. Figure 1 shows size distribution and TEM images of UA-PTX-LiP which reveal its size (about $150 \mathrm{~nm}$ ). This was in line with the results obtained from DLS analysis. The discrepancies in sizes were due to that fact that TEM image of UA-PTX-LiP was acquired in the dry state under vacuum, whereas the DLS profile was obtained in an aqueous dispersion.

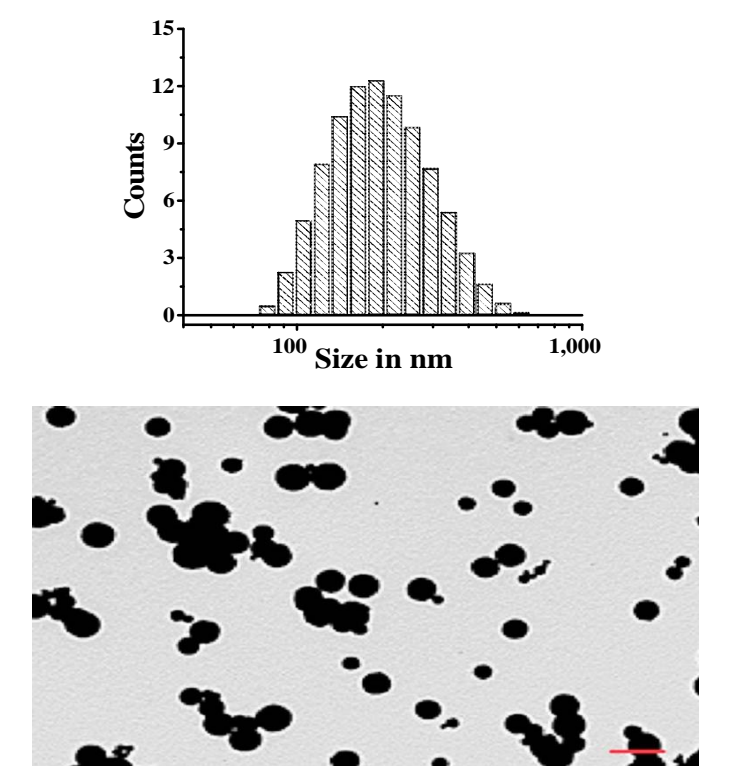

Figure 1: Size distribution of UA-PTX-LiP and its transmission electron micrograph. The scale bar represents the scale of $200 \mathrm{~nm}$

\section{Cytotoxicity}

Free PTX, taxol, PTX-LiP and UA-PTX-LiP dosedependently induced cytotoxicity in HSC-3 cancer cells (Figure 2). In particular, UA-PTX-LiP showed higher cytotoxic effect than pure PTX or PTX-LiP at all tested concentrations. At a ratio of 1:1, PTX and UA were significantly more cytotoxic $\left(\mathrm{IC}_{50}=0.2 \mu \mathrm{g} / \mathrm{mL}\right)$ than pure PTX at a lower dose. These results (including those from cellular uptake studies) establish that UA-PTX$\mathrm{LiP}$ is effective in causing more accumulation of intracellular drugs through endocytosis, resulting in greater ability to activate caspase- 3 and induce more cell apoptosis [22]. They also indicate the enhancement of anticancer activity through ratiometric loading of PTX and UA, relative to single drugs.

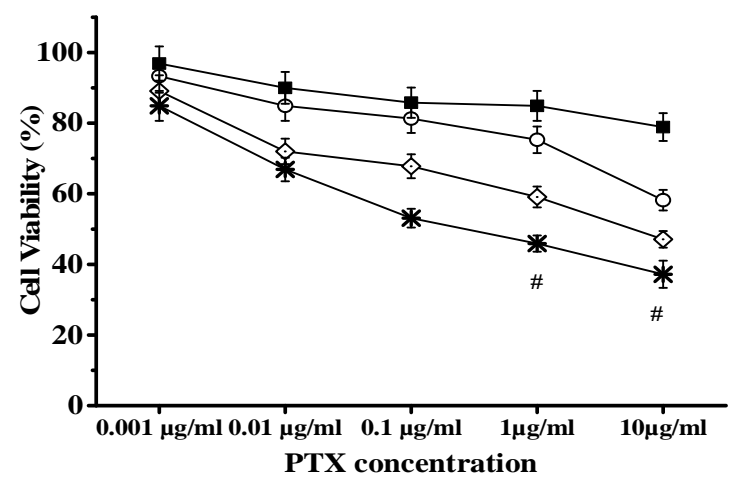

Figure 2: Cell viability of HSC-3cells exposed to $0.001,0.01,0.1,1$ and $10 \mu \mathrm{g} / \mathrm{ml}$ of PTX, taxol, PTXLiP and UA-PTX-LiP ( $n=6, \# p<0.05)$. ( $\longrightarrow$ PTX, $\multimap-$ Taxol, $\prec-$ PTX-LiP, $\because$ UA-PTX-LiP) 


\section{Apoptosis}

The results obtained from double staining assay using Annexin V/PI with respect to the effects of UA-PTX-LiP, PTX-LiP and control on apoptosis in HSC-3 cell lines are shown in Figure 3. The apoptotic cells were more in cells treated with UA-PTX-LiP than in cells treated with PTX-LiP or control. Thus, UA-PTX-LiP induced apoptosis specifically in HSC-3 cell lines.
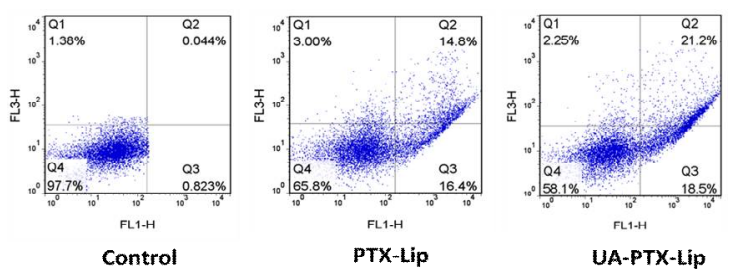

Figure 3: Effect of PTX-LiP and UA-PTX-LiP on apoptosis in HSC-3 cells, as determined using double staining with FITC-Annexin V/PI. Apoptosis induction in HSC-3 was observed in PTX-LiP (14.8\%) and UAPTX-LiP (21.2\%) after the treatment. The graph was plotted between FL-1 and FL-3 representing Annexin $\mathrm{V}$ and $\mathrm{PI}$ positive cells, respectively. UA-PTX-LiP exhibited significantly $(p<0.05)$ higher apoptotic capacity in HSC-3 cells than PTX-LiP

\section{Intracellular reactive oxygen species (ROS)}

The results obtained showed that UA enhanced ROS levels in HSC-3 cells, but UA-PTX-LiP produced highly ROS in HSC-3 cells than UA. Intracellular ROS accumulation by LiP could be one of the key factors involved in induction of apoptosis in cancer cells. Oxidative stress is caused by direct ROS accumulation in cancer cells or inhibition of endogenous antioxidants. Thus, UA manipulates the redox state of cancer cells by initiating intracellular ROS, thereby leading to cellular apoptosis [23].

\section{Effect of treatments on LDH levels}

The pattern of $\mathrm{LDH}$ release after cell treatment using PTX, UA-PTX-LiP and PTX-LiP is presented in Figure 4. The release of $L D H$ due to UA-PTX-LiP treatment was minimal.

\section{Effect of treatments on cellular uptake of PTX}

The cellular uptakes of the control, PTX-LiP, and UA-PTX-LiP were evaluated with flow cytometer.

Green fluorescence arising from FITC was used to estimate uptake from the liposome. As revealed in Figure 6, all LiPs produced significant cellular uptake in HSC-3 cells. However, UAPTX-LiP showed a surprisingly higher uptake in HSC-3 cells, when compared to PTX-LiP and control. Cells in general can take up liposomes non-specifically via the general biochemical reactions with the receptors or via endocytosis [24]. High cellular internalization of UA-PTX-LiP may be due to the presence of UA [25].

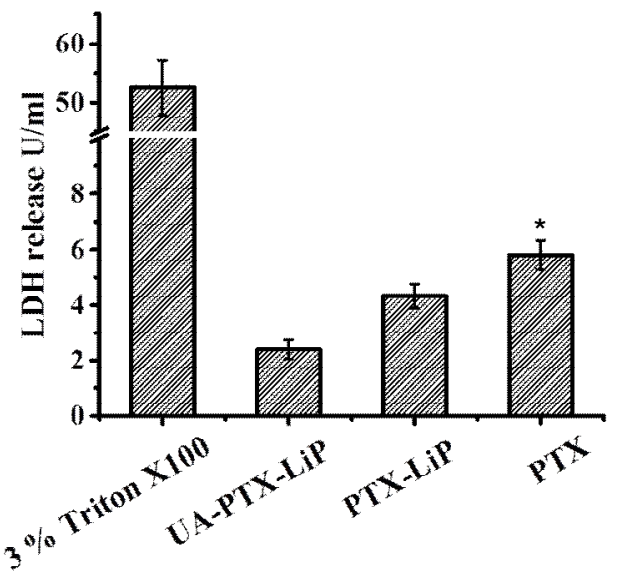

Figure 4: $\mathrm{LDH}$ release from $\mathrm{HSC}-3$ cells after treatment with UA-PTX-LiP, PTX-LiP and PTX. The positive control was $3 \%$ Triton-X100. Results are shown as mean \pm SD $(\mathrm{n}=3)$. ${ }^{*} p<0.05$ : PTX compared with PTX-Lip and UA-PTX-Lip

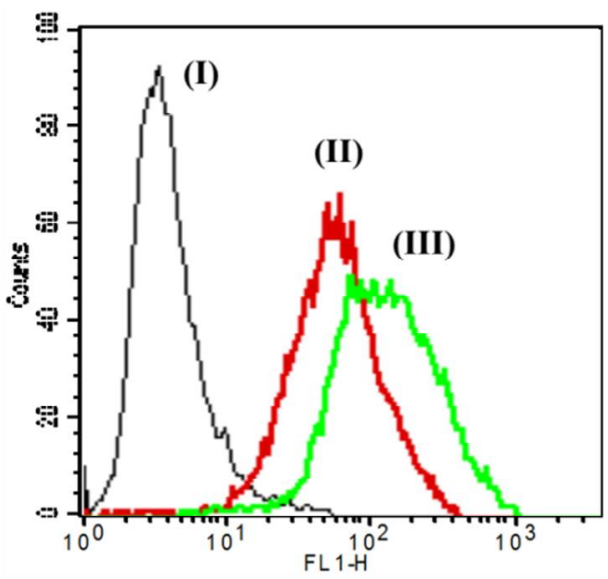

Figure 6: Effect of treatments on uptake in HSC-3 cells, determined using flow cytometry. The $X$ axis represents fluorescence within the cell. Fluorescence was shown in (i) control (ii) FITC-LiP, (iii) UA-FITC-LiP

\section{DISCUSSION}

Even after being continuous progress in the diagnosis, chemotherapy and adjuvant therapies for HNSCC, toxicity remained an urgent issue for patients with HNSCC. PTX is widely used as an anticancer drugs for various cancer including HNSCC, but has been associated with severe toxicity [26]. The combination of different chemotherapeutics is of great advantage; especially the herbal combination can provide reduced toxicity and enhance anticancer activity [27]. Here we report the liposomal co-delivery of PTX and UA with the aim to reduce the toxicity 
and increase the potency of PTX. The functionality of the nanotechnology has been exploited to load UA and PTX. The particle size of UA-PTX-LiP measured using DLS was slightly higher than that of measured by TEM which might be attributed to the aqueous dispersion of the liposomes measured by DLS. Further, we evaluated the in-vitro efficacy of UA-PTX-LiP in HSC-3 cell lines for their potency in killing the cells and also the mechanism of its enhanced activity was also established. The results suggests that liposomal drug delivery system (UA-PTX-LiP) show significant advantages over free UA and PTX in assessing in vitro cellular uptake through improved drug diffusion in the cells [28].

PTX and UA combination was significantly more cytotoxic than PTX alone at lower doses. In vitro antitumor evaluation, i.e., FACS, immunofluorescence assay and apoptosis indicated that UA-PTX-LiP inhibited the growth of head and neck cancer cells and increased cellular uptake which induced cellular apoptosis more powerfully than PTX-LiP and free PTX. UAPTX-LiP was capable of inducing apoptosis greater than PTX-LiP, which might be attributed to the presence of UA which have inhibited COX2 and induced apoptosis [21]. It was also observed that UA-PTX-LiP showed higher potency than PTX-LiP and free PTX as UA exerted increased oxidative effect on HSC-3 cells which facilitates its cytotoxic effect, our results were consistent with previous research [29]. Furthermore, the results show that UA a natural triterpenoid have a significant effect in chemosensitizing HSC-3 cells. UA-PTX-LiP could provide a more potent combination than PTX alone as indicated in in-vitro experiments. It will be of great value if further studies including the animal model could be done to establish the actual efficacy of the combination therapy.

\section{CONCLUSION}

PTX and UA co-loaded liposomes has been successfully developed and evaluated for their anticancer potency. UA-PTX-LiP is more cytotoxic than PTX alone even at low concentrations. Therefore, UA-PTX-LiP is a potential combination system for cancer therapy, especially head and neck cancer tumors.

\section{DECLARATIONS}

\section{Acknowledgement}

This project was supported by Funding of Health and Family Planning Commission of Hubei Province (nos. WJ2017M174 and WJ2017M240).

\section{Conflict of interest}

No conflict of interest is associated with this work.

\section{Contribution of authors}

We declare that this work was done by the authors named in this article and all liabilities pertaining to claims relating to the content of this article will be borne by the authors. Bi-Hua Lv and Wei Tan contributed equally to this work. The project work was designed by Bi-Hua Lv, Ling Zhang. The experiments were carried out by Bi-Hua Lv, Wei Tan, Xuejun Shang. The manuscript writing and editing was carried out by Bi-Hua Lv, Wei Tan, Xuejun Shang, Ling Zhang.

\section{REFERENCES}

1. Wu TT and Zhou SH. Nanoparticle-Based Targeted Therapeutics in Head-And-Neck Cancer. Int J Med S 2015; 12(2): 187-200.

2. Gligorov $J$ and Lotz JP. Preclinical pharmacology of the taxanes: implications of the differences. Oncologist 2004; (9 Suppl) 2: 3-8.

3. Guastalla JP, Lhomme C, Dauplat J, Namer $M$, Bonneterre J, Oberling $F$, Pouillart $P$, Fumoleau $P$, Kerbrat $P$, Tubiana $N$, et al. Taxol (paclitaxel) safety in patients with platinum pretreated ovarian carcinoma: an interim analysis of a phase II multicenter study. Ann Oncol 1994; (5 Suppl) 6: S33-38.

4. Banno N, Akihisa T, Tokuda H, Yasukawa K, Higashihara $H$, Ukiya $M$, Watanabe $K$, Kimura $Y$, Hasegawa $J$ and Nishino $H$. Triterpene acids from the leaves of Perilla frutescens and their anti-inflammatory and antitumorpromoting effects. Biosci Biotech Bioch 2004 Jan; 68(1): 85-90.

5. Zhang $Y$, Mintzer $E$ and Uhrich KE. Synthesis and characterization of PEGylated bolaamphiphiles with enhanced retention in liposomes. J Colloid Interface Sci 2016; 482: 19-26.

6. Dwivedi $P$, Khatik $R$, Khandelwal $K$, Shukla $R$, Paliwal SK, Dwivedi $A K$ and Mishra PR. Preparation and characterization of solid lipid nanoparticles of antimalarial drug artemether for oral administration. $J$ Biomater Tiss Eng 2014; 4(2): 133-137.

7. Khandelwal K, Pachauri SD, Arya A, Pawar VK, Joshi T, Dwivedi $P$, Ahmad $H$, Singh B, Sharma K, Kanojiya S, et al. Improved oral bioavailability of novel antithrombotic S002-333 via chitosan coated liposomes: a pharmacokinetic assessment. RSC Adv 2015; 5(49): 39168-39176.

8. Gupta PK, Jaiswal AK, Kumar V, Verma A, Dwivedi $P$, Dube $A$ and Mishra PR. Covalent functionalized selfassembled lipo-polymerosome bearing amphotericin $B$ for better management of leishmaniasis and its toxicity evaluation. Mol Pharm 2014; 11(3): 951-963.

Trop J Pharm Res, November 2018; 17(11): 2120 
9. Ruttala HB and Ko YT. Liposome encapsulated albuminpaclitaxel nanoparticle for enhanced antitumor efficacy. Pharm Res 2015; 32(3): 1002-1016.

10. Harmand PO, Duval R, Delage $C$ and Simon A. Ursolic acid induces apoptosis through mitochondrial intrinsic pathway and caspase-3 activation in M4Beu melanoma cells. Int J Cancer 2005; 114(1): 1-11.

11. Achiwa $Y$, Hasegawa $K$, Komiya $T$ and Udagawa $Y$. Ursolic acid induces Bax-dependent apoptosis through the caspase-3 pathway in endometrial cancer SNG-II cells. Oncol Rep 2005; 13(1): 51-57.

12. Shanmugam MK, Ong TH, Kumar AP, Lun CK, Ho PC, Wong PT, Hui KM and Sethi G. Ursolic acid inhibits the initiation, progression of prostate cancer and prolongs the survival of TRAMP mice by modulating proinflammatory pathways. PloS one 2012; 7(3): e32476.

13. Wang T, Petrenko VA and Torchilin VP. Paclitaxel-loaded polymeric micelles modified with MCF-7 cell-specific phage protein: enhanced binding to target cancer cells and increased cytotoxicity. Mol Pharm 2010 Aug 2; 7(4): 1007-1014.

14. Akbarzadeh A, Rezaei-Sadabady R, Davaran S, Joo SW, Zarghami N, Hanifehpour $Y$, Samiei $M$, Kouhi $M$ and Nejati-Koshki K. Liposome: classification, preparation, and applications. Nanoscale Res Lett 2013; 8(1): 102102.

15. Tripathi $P$, Verma $A$, Dwivedi $P$, Sharma $D$, Kumar $V$, Shukla $R$, Banala VT, Pandey G, Pachauri $S D$ and Singh SK. Formulation and characterization of amphotericin b loaded nanostructured lipid carriers using microfluidizer. J Biomater Tiss Eng 2014; 4(3): 194-197.

16. Li X, Li R, Qian X, Ding Y, Tu Y, Guo R, Hu Y, Jiang X, Guo $W$ and Liu B. Superior antitumor efficiency of cisplatin-loaded nanoparticles by intratumoral delivery with decreased tumor metabolism rate. Eur $J$ Pharm Biopharm 2008; 70(3): 726-734.

17. Tsai YM, Chien CF, Lin LC and Tsai TH. Curcumin and its nano-formulation: the kinetics of tissue distribution and blood-brain barrier penetration. Int J Pharm 2011; 416(1): 331-338.

18. Yang G, Yang T, Zhang W, Lu M, Ma X and Xiang G. In Vitro and in Vivo Antitumor Effects of Folate-Targeted Ursolic Acid Stealth Liposome. J Agr Food Chem 2014; 62(10): 2207-2215.

19. Song $C K$, Jung $S H$, Kim $D D$, Jeong $K S$, Shin $B C$ and Seong $H$. Disaccharide-modified liposomes and their in vitro intracellular uptake. Int J Pharm 2009; 380(1-2): 161-169.

20. Lin $Y$, Fujimori T, Kawaguchi N, Tsujimoto $Y$, Nishimi $M$, Dong $Z$, Katsumi $H$, Sakane $T$ and Yamamoto $A$.
Polyamidoamine dendrimers as novel potential absorption enhancers for improving the small intestinal absorption of poorly absorbable drugs in rats. J Control Release 2011; 149(1): 21-28.

21. Zhang H, Li X, Ding J, Xu H, Dai X, Hou Z, Zhang K, Sun $K$ and Sun $W$. Delivery of ursolic acid (UA) in polymeric nanoparticles effectively promotes the apoptosis of gastric cancer cells through enhanced inhibition of cyclooxygenase 2 (COX-2). Int J Pharm 2013 Jan 30; 441(1-2): 261-268.

22. Ramasamy T, Haidar ZS, Tran TH, Choi JY, Jeong J-H, Shin BS, Choi H-G, Yong CS and Kim JO. Layer-bylayer assembly of liposomal nanoparticles with PEGylated polyelectrolytes enhances systemic delivery of multiple anticancer drugs. Acta Biomater 2014; 10(12): 5116-5127.

23. Xu H, Li X, Ding W, Zeng X, Kong $H$, Wang $H$ and Xie W. Deguelin induces the apoptosis of lung cancer cells through regulating a ROS driven Akt pathway. Cancer Cell Int 2015; 15: 25.

24. Osaka T, Nakanishi T, Shanmugam S, Takahama $S$ and Zhang $H$. Effect of surface charge of magnetite nanoparticles on their internalization into breast cancer and umbilical vein endothelial cells. Colloids Surf $B$ Biointerfaces 2009; 71(2): 325-330.

25. Chen B, Le W, Wang Y, Li Z, Wang D, Ren L, Lin L, Cui $S$, Hu JJ, Hu Y, et al. Targeting Negative Surface Charges of Cancer Cells by Multifunctional Nanoprobes. Theranostics 2016; 6(11): 1887-1898.

26. Citrin D, Mansueti J, Likhacheva A, Sciuto L, Albert PS, Rudy SF, Cooley-Zgela $T$, Cotrim A, Solomon $B$, Colevas $A D$, et al. Long-Term Outcomes and Toxicity of Concurrent Paclitaxel and Radiation in the Treatment of Locally Advanced Head and Neck Cancer. Int J Radiat Oncol Biol Phys 2009; 74(4): 1040-1046.

27. Xu X, Zhu G-Q, Zhang K, Zhou Y-C, Li X-L, Xu W, Zhang $H$, Shao $Y$, Zhang $Z-Y$ and Sun $W-H$. Cyclooxygenase-2 mediated synergistic effect of ursolic acid in combination with paclitaxel against human gastric carcinoma. Oncotarget 2017; 8(54): 92770-92777.

28. Gupta PK, Asthana S, Jaiswal AK, Kumar V, Verma AK, Shukla $P$, Dwivedi $P$, Dube $A$ and Mishra $P R$. Exploitation of lectinized lipo-polymerosome encapsulated Amphotericin B to target macrophages for effective chemotherapy of visceral leishmaniasis. Bioconjug Chem 2014; 25(6): 1091-1102.

29. Shen S, Zhang Y, Zhang R, Tu $X$ and Gong $X$. Ursolic acid induces autophagy in U87MG cells via ROSdependent endoplasmic reticulum stress. ChemicoBiological Interactions 2014 2014/07/25/; 218: 28-41. 